\title{
Survey on Vehicle Detection and Tracking Techniques in Video Surveillance
}

\author{
Swathy M. \\ M-Tech Student \\ Department of CSE \\ APJ Abdul Kalam \\ Technological University
}

\author{
Nirmala P. S. \\ M-Tech Student \\ Department of CSE \\ APJ Abdul Kalam \\ Technological University
}

\author{
Geethu P. C. \\ Assistant Professor \\ Department of CSE
}

\begin{abstract}
It is important to know the road traffic density for effective traffic management and intelligent transportation system (ITS). The increasing traffic volume creates a greatest challenge in today's traffic research. This work is to detecting moving vehicles in video streams of traffic scenes recorded by low resolution cameras using some of the image processing techniques. Vision based traffic surveillance is a fast emerging field in road management schemes and highway monitoring. Video cameras are used to provide a rich information source for human understanding. Video sequences are captured and tested with the image processing techniques. Many methods and algorithms have been proposed in this paper to detect vehicles on highways..
\end{abstract}

\section{General Terms}

Image processing Intelligent Transportation system,Low resolution cameras

\section{Keywords}

Image processing techniques,Traffic Analysis, Vehicle detection, Vehicle tracking.

\section{INTRODUCTION}

The most important applications of vision based supervision systems is the traffic surveillance. For many years the researches performs more works in the Vision-Based Intelligent Transportation System (ITS), traffic engineering applications and transportation planning to extract exact traffic information for traffic image analysis and traffic flow control like vehicle count, vehicle velocity, traffic lane changes, vehicle trajectory, vehicle classification, traffic density, vehicle tracking,vehicle flow, license plate recognition, etc. . In the past, the vehicle segmentation and detection, tracking systems used to determine the charge for various kinds of vehicles for automation toll levy system. Recently, vehicle recognition system is used to detect (the vehicles) or detect the traffic lanes or classify the type of vehicle class on highway roads like cars, motorbikes, vans, heavy goods vehicles (HGVs), buses and etc.

Increasingly traffic levels and busier roads are most common across the globe. Consequently, there is an need to develop intelligent traffic surveillance systems that play an important role in highway monitoring and road management systems. Their purpose is to provide real-time numerical data on traffic activity and to signal potentially abnormal situations, e.g., accident detection or dangerous driving.

Intelligent image detection systems are most important approach to modern day traffic management. This has arisen for the need of most cost effective and efficient traffic monitoring. In addition, it increases the choice for automatic analysis of urban traffic activity from CCTV in recent years.
This increase contributed to the additional numbers of cameras and other sensors, improved infrastructure and subsequent accessibility of data. The advancement of analytical techniques to process the video together with increased computing power has enabled new applications. Video analytics is defined as surveillance based computer vision algorithms and systems to extract appropriate information from video. The main concept is to support human operators in observing video data. This can allow postevent detection of events of interest, which is useful for traffic management due to additional data available. Automatic preprocessing allows efficient control for the operators to pick cameras to view and collect statistics, with the aim to improve traffic flow. Video cameras have been used for a long time for traffic monitoring purposes, because they provide a rich information for human understanding.

This paper focus on different image processing techniques that can detect and track the vehicles for better traffic surveillance. We carry out various tasks such as foreground segmentation, feature extraction,background subtraction and Thresholding techniques

\section{RELATED WORKS}

Raad Ahmed Hadi, Ghazali Sulong and Loay Edwar Georg [1] proposed the paper "vehicle detection and tracking techniques: a concise review" This paper focuses on the Vehicle tracking and detection play an important role in civilian and military applications such as in management and urban traffic planning ,highway traffic surveillance control. Vehicle detection process on road are used for counts, average speed of each individual vehicle tracking, traffic analysis and vehicle categorizing objectives and may be implemented under different environments changes. In this, we present a concise overview of different image processing methods and analysis tools used in building these applications that developing traffic surveillance systems. More precisely and in compare with other reviews, we classified the processing methods into three categories to explain the traffic systems.

V. Kastrinaki, M. Zervakis, K. Kalaitzakis [2] proposed the paper" A survey of video processing techniques for traffic applications" Video sensors plays important role in traffic applications mainly due to their easy installation, operation and maintenance ,fast response, and their ability to monitor wide areas. Research in traffic applications has results in a most of video processing and analysis methods. Two of the most challenging and widely studied applications related to traffic monitoring and automatic vehicle guidance. In general, systems developed for the areas must integrate, amongst the other tasks, the analysis of the static environment (automatic lane finding) and the detection of static or moving obstacles (object detection) within the space of interest. In this paper we present an outline of image processing and analysis tools used 
in these applications and relate these tools with most complete systems developed for particular traffic applications. More specifically, categorize processing methods based on the fundamental organization of their input data (feature-driven, area-driven, or model-based) and the domain of processing (spatial/frame or temporal/video). Furthermore, we differentiate between the cases of static and mobile camera. Based on this categorization of processing tools, we present representative systems that have been used for operation. Thus, the purpose of the paper is threefold. First, to classify image-processing methods used in traffic applications. Second, to provide the advantages and disadvantages of the algorithms. Third, from this both consideration,is an attempt to evaluation of shortcomings and general needs in this field of active research.

Naveen Chintala cheruvu, Venkatesan Muthukumar[3] proposed the paper "Video Based Vehicle Detection and Its Application in Intelligent Transportation Systems" Video based vehicle detection technology is the most important part of Intelligent Transportation System (ITS), due to its nonintrusiveness and inclusive vehicle behavior data collection capabilities. This paper introduces an efficient vehicle detection system based on Harris-Stephen corner detector algorithm. The algorithm was used to develop a vehicle detection and tracking system that determines vehicle counts and speeds at road- ways and freeways. The proposed video surveillance based vehicle detection system was developed to eliminate the need of complex setting, robustness to different variations, and perform better with low resolutions videos. The algorithm performance for accuracy in vehicle counts and speed was evaluated. The performance of the proposed system is equivalent compared to other commercial vehicle detection system. Using the developed vehicle detection and tracking system an advance warning intelligent transportation system was designed and implemented to alert computers in advance of speed reductions and congestions at area zones and special events.

Siddhartha A. Meshram, Prof. Vishal. B. Raskar[4]proposed the paper "vehicle detection and tracking techniques used in moving vehicles" The difficulty of obtaining the initial background there is the inaccuracy of real-time background update and the difficulty of controlling the update speed in moving vehicle detection of traffic video this paper proposes an accurate and effective moving vehicle detection method used in complex traffic environment. This method first constructs initial background image according to the real-time situation of traffic environment.Then it segmentises the current frame into foreground region and background region accurately using the combined method of subtraction method and inter-frame difference. The results show that method can detect moving vehicles fast and accurately in traffic situation. Vehicle detection and tracking applications play a main role for civilian and military applications such as in highway traffic surveillance control management.

Antoni B. Chan, Member, IEEE, and Nuno Vas concelos[5] proposed the paper" Counting People With Low-Level Features and Bayesian Regression" An approach to the problem of estimating the size of in similar crowds, which are composed of pedestrians that travel in different directions, without using explicit object segmentation or tracking is proposed. Instead, the crowd is segmented into components of homogeneous motion, using mixture of dynamic-texture motion model. A set of low-level features is extracted from each segmented region, and function that maps features into number of people per segment is learned with Bayesian regression. Two Bayesian regression models are examined. The rest is a mixture of Gaussian process regression with a compound kernel, that accounts for both the local and global trends of the count mapping but is limited by the real valued outputs that do not match the discrete counts. Here this model is limitation is addressed with a second model, that is based on a Bayesian treatment of Poisson regression that introduces a preceding distribution on the linear weights of the model. Since exact interpretation is systematically inflexible, a closed-form approximation is derived that is computationally efficient and kernelizable,enabling there presentation of nonlinear functions. An approximate marginal likelihood is also derived for kernel hyper parameter learning.

Abinaya,K.S.Ravichandran,B.Santhi[6] proposed the paper "Watershed Segmentation for Vehicle Classification and Counting" - A robust video based system for the traffic surveillance system on the highway for vehicle detection, vehicle classification and counting for effective traffic analysis using only a single standard camera. The key goal of the proposed work is to successfully detect, track vehicle in partial occlusion and connected together by shadow on the highways. Marker-controlled watershed segmentation method is initially used for the extraction of the foreground regions from the highway scene. For tracking Gabor filter is applied used to measure vehicle path in video sequences. Support vector machine is utilized for effective vehicle classification. A result shows the considerable performance of watershed segmentation in vehicle detection and connected together by shadow in the highways environment.

Guohui Zhang ,Ryan P. Avery[7] proposed the paper" A Video-based Vehicle Detection and Classification System for Real-time Traffic Data Collection Using Uncalibrated Video Cameras"Length-based vehicle classification data are inputs for pavement design, and transportation planning, traffic operation. However, such data are not measurable by single loop detectors, the most widely deployed type of traffic sensor in the existing roadway infrastructure. In this study a Videobased Vehicle Detection and Classification (VVDC) system was developed for truck data collection.

\section{ELEMENTS OF TRAFFIC ANALYSIS SYSTEM 3.1 Vehicle Detection}

\subsubsection{Object based approach:}

This approach has three steps for detecting the vehicles 1.segmentation 2.training 3.validation. In first step it takes some training images from total number of images depending upon number of frames. Then it proceed with the optimization of the segmentation parameters for segmentation and is repeated for training samples that involve multi resolution segmentation and the spectral difference segmentation and then it transfer to validation part of object accuracy valuation with the training samples[3]. In this model the condition of applicability to high spatial resolution weakly sensed data, and to address the essential for a quantitative, user-supervised method for taking best segmentation parameters. It developed an impartial metric which is the number of training object matched with maximize area matched and is minimizes below and over segmentation for chosen images in objective primitives.

\subsubsection{Background Subtraction Methods}

Background subtraction also known as foreground detection is a techniques in fields of image processing wherein an image's foreground is extracted for extra processing. 
Background subtraction is the most widely used approach for detecting moving objects in videos from static cameras. It is mostly done if the image is a part of a video stream[5].

In Background Subtraction, the variation of the intensity values of the background pixel are done with the following methods, such as non-parametric Kernel Density Estimation, Mixture of Gaussians Unimodal distributions and Adaptive multi-cue Background Subtraction. In Background Subtraction algorithm, the foreground vehicles are separated from the background and form a foreground mask. Background Subtraction is used in object tracking, video appliances, traffic analysis, target tracking and also used to detect foreground object by comparing the different frames. If the threshold value is less than the difference image, then it is taken as a moving object or otherwise taken as a background image. The video sequences are observed in such a way that, I is made of a static background $\mathrm{B}$, that it observes every moving object is made of a color and here the Background Subtraction encapsulate the formula such as ;

$$
X t(s)=1 \text { if } d(\mathrm{Is}, t, \mathrm{Bs})>t,
$$

otherwise 0 Where $X t$ is the motion label field at time $\mathrm{t}, \mathrm{t}$ is a Threshold, and Bs,the background model at pixel s, $\mathrm{d}$ is the distance between Is,t,the color at time $t$ and pixel $s$. This technique is based on computing the error between the background frame and current frame. Some of the background techniques are:

\subsubsection{Gaussian Mixture Model (GMM)::}

Each pixel is assumed to be a mixture of Gaussian distribution in mixture of Gaussian model[9]. The Gaussian Mixture Model are sampled and expected as diagonal, spherical, tied and full covariance matrices supported from data. It provides the number of components properly. From a mixture of a finite number of Gaussian distributions with the unknown parameters,ie the probabilistic model, which assumes all the data points, where Gaussian mixture model is a probabilistic model. It generates $\mathrm{k}$-means clustering to incorporate the covariance structure of data and centers of latent Gaussians. For learning mixture models, Gaussian Mixture Model is the fastest algorithm. If it has many points per mixture, which is inadequate, so estimating the covariance matrices suits difficult. In the absence of hints, only the data theoretical criterion decides how many components are to be used.

\subsubsection{Adaptive multi-cue background subtraction:}

In Adaptive multi-cue background subtraction, this technique is to obtain correct background subtraction results by using different hints. This technique proven to be the best approach. There are two serious issues are: (i) which way cues are merged, and (ii) which cues are to be used. Pixel intensity, pixel color and edges are the useful. In the measurement of traffic data, the approaches offer the exciting conclusions namely, vehicle counting and classification. Here, the background is continuously trained when the system observes the scene, and is adapted to global changes, and it affects the background mean and standard deviation values. Image processing methods that is related to the resolution and frame rate requires some of the computation power. Here, new multi-cue segmentation architecture is used to arrange different image cues, it combines both the bottom-up and topdown strategies to solve local and global illumination changes and obtain a background model. The bottom-up strategy includes a higher sensitivity and a color model to scene changes using conical and cylindrical RGB models and gradient cues, which support segmentation masks efficiently. According to its chromaticity and luminance characteristics, different segmented regions are easily defined, whereas in thetop-down strategies, the scene information are taking from shadows. In sunny days and also in dense traffic situations, the improved background model is used and ignores the moving objects. Here, the proposed background subtraction method describes to perform well, to obtain the volume and position for estimating the vehicles 2D/3D strategy heavily depend on it. Real-time performance can be achieved by implementing this algorithm.

\subsubsection{Averaging}

In the background averaging method, all video frames are added up[7]. The learning rate requires the weight between a new frame and the background. This algorithm has little computational cost, however, it is likely to produce the tails behind moving objects due to contamination of the background with the appearance of the moving objects.Use the prompt background, which is the current frame with detected objects removed. The regions of detected objects are filled with the old background pattern. By averaging the instantaneous background, the tails generated by moving objects are reduced. The feedback of the motion mask could lead to erroneous background estimations, if the threshold is set poorly. A dynamic threshold is applied to reduce this problem of never updating a region detected as foreground.

\subsubsection{Kalman Filter}

A Kalman filter can be used to estimate the background image, in this the colour of each pixel is modelled by one filter. The foreground can be taken as noise for the filter state. However, illumination changes are non Gaussian noise and disrupt assumptions for the use of Kalman filters.

\subsubsection{Feature Based Methods}

Another trend which the researchers investigate on subfeatures like the corners and edges of vehicles, the moving objects segmented from background image by collecting the set of these features from the movement between the subsequent frames. The feature based method supports the occlusion handling between the overlapping vehicles and then it compared with background subtraction method represents a less level from the computation.

Several approaches can distinguish the object from the background by using its features. This approach based on learning which employs a set of labeled training data used for labeling the extracted objects features. In addition, it uses a Haar wavelets technique for feature extraction method and uses support vector machine classifier for classification process. Additionally, face, people and car images datasets have tested on this approach.

\subsubsection{Motion Based Methods}

Optical flow is an approximation of the image motion on the basis of local derivatives in a given sequence of images. That is, it specifies how much each image pixel moves between adjacent images. Although most optical flow-based techniques require high computational effort, object recognition via background subtraction techniques usually require significantly lower computational effort.

\subsection{Vehicle Tracking}

Tracking of vehicle is the process of monitoring the location of a bus, car, truck or any moving vehicle using the GPS system. Widely most deployed to keep track of truck fleets, vehicle tracking ensures that the vehicles are used properly 
and that can be recovered in the event they are taken or stolen. Multi-object tracking and data association have received huge attention in the computer vision field and much of the background work has been done in the non-transportation applications There are some object tracking methods in vehicle tracking:

\section{Region-Based Tracking Methods \\ 2. Contour Tracking Methods \\ 3. 3D Model-Based Tracking Methods \\ 4. Feature-Based Tracking Methods \\ 5. Color and Pattern-Based Methods}

\subsubsection{Region based tracking Methods:}

In this approach, it identifies a connected region in the image, ie. 'blob', associated with each vehicle and then it tracks it over the time using a cross-correlation measure. Typically, this process is initialized by the background subtraction technique[1]. A Kalman filter-based adaptive background model makes the background estimate to evolve as the weather and time of day might affect the lighting conditions.

This approach works properly well in free-flowing traffic. However, under congested traffic conditions, the vehicles partially occlude one another instead of being spatially isolated, that makes the task of segmenting individual vehicles difficult. Such vehicles will become grouped together as large blob in the foreground image

\subsubsection{Contour Tracking Methods:}

The dual to region based approach is tracking based on active contour models. The basic idea is to make a representation of the bounding contour of the object and keep on dynamically updating it. The advantage of a contour based representation instead of a region based representation is reduced computational complexity.

\subsubsection{Model-Based Tracking Methods}

The occlusion of vehicles detection used a 3D solid cuboid form with up to six vertices, and this cuboid used to fit many different types and sizes of vehicle images by varying the vertices for a best fit.

A new 3D model-based vehicle detection framework is based on a probabilistic boundary feature grouping, used for vehicle tracking and detection.This framework supported advantageous characteristics such as the flexibility due to it is free from the scale problem and detects the vehicles from more diagonal visions.It is fast when applied to many other applications.

\subsubsection{Feature-Based Tracking Methods}

An iterative and different framework based on edge points and features as modified SIFT descriptors used in similarity process, these features represents a large set of features forms a strong interpretation for object classes[2]. The proposed framework showed a good performance for the vehicle classification in surveillance videos regardless of significant challenges such as quality, limited image size and and large intra-class dissimilarities.

\subsubsection{Color and Pattern-Based Methods}

It uses the above technique by encompassed the $\mathrm{YCrCb}$ color space for the construction preliminary background, vehicle location, vehicle tracking, shade elimination,segmenting foreground, and background updating algorithms. Through the experiments, this system proves that it work under several weather conditions, and is insensitive to lighting.A modelbased system for traffic supervision visual tracing and classification of vehicles for multi-lane highway scene.

\section{CONCLUSION}

In this paper,presents different vehicle tracking and detection techniques on surveillance videos. Although there are many sensors, it possess high maintainence cost. Additionally, this system differentiates itself from other computer-vision-based approaches in such a way that can handle the system without the need for any hardware other than cameras. This makes the system inexpensive to use.These types shows the detailed information about how the traffic surveillance systems used the image processing methods and analysis tools for detect, segment, and track the vehicles.

\section{ACKNOWLEDGMENT}

We would like to thank the Lord Almighty, the foundation of all wisdom who has been guiding us in every step. We wish to record our indebtedness and thankfulness to all who helped us to provide information and necessary facilities. We would like to thank the guide Ms Beena M V, Assistant Professor in the Department of Computer Science And Engineering, for valuable guidance. Our sincere thanks to parents for their blessings and colleagues for their help.

\section{REFERENCES}

[1] Benjamin Coifmana,,David Beymerb, Philip McLauchlanb, Jitendra Malik, "A real-time computer vision system for vehicle tracking and traffic surveillance," Transportation Research Part C 6 (1998) $271 \pm 288$

[2] Siddhartha A. Meshram, Prof. Vishal. B. Raskar,"vehicle detection and tracking techniques used in moving vehicle," International Journal of Innovative Science, Engineering \& Technology, Vol. 2 Issue 7, July 2015.

[3] Naveen Chintalacheruvu, Venkatesan Muthukumar, "Video Based Vehicle Detection and Its Application in Intelligent Transportation Systems," Journal of Transportation Technologies, 2012, 2, 305-314

[4] V. Kastrinaki, M. Zervakis, K. Kalaitzakis," A survey of video processing techniques for traffic applications," Image and Vision Computing 21 (2003) 359-381

[5] Raad Ahmed Hadi1, Ghazali Sulong , Loay Edwar George," vehicle detection and tracking techniques: a concise review," Signal \& Image Processing : An International Journal (SIPIJ) Vol.5, No.1, February 2014

[6] Susmita A. Meshram,A.V.Malviya," Traffic Surveillance by Counting and Classification of Vehicles from Video using Image Processing," Volume 1, Issue 6, November 2013

[7] G. Poorani, K. Dhana Shree, Dr. A. Grace Selvarani,” A Survey on Counting and Classification of Highway Vehicles," Volume 5, Issue 9, September 2015

[8] Tsuyoshi Idé, Takayuki Katsuki, Tetsuro Morimura," Monitoring Entire-City Traffic using Low-Resolution Web Cameras"

[9] Mingpei Liang, Xinyu Huang, Chung-Hao Chen, Xin Chen, and Alade Tokuta, "Counting and Classification of Highway Vehicles by Regression Analysis", IEEE transactions on intelligenttransportationsystems, vol. 16, no. 5 , october 2015 